\title{
Análisis documental de contenido de textos narrativos: bases epistemológicas y perspectivas metodológicas
}

Documentary content analysis of narrative texts:

epistemological backgrounds and methodological perspectives

\author{
José Augusto Chaves Guimarães (1), João Batista Ernesto de Moraes (2) \\ y Maura Duarte Moreira Guarido (3)
}

(1) Faculdade de Filosofia e Ciências - Unesp, Av. Higinio Muzzi Filho, 737 Marília, São Paulo, Brasil CEP: 17525-900, guimajac@marilia.unesp.br. (2) jota@marilia.unesp.br. (3) mauraguarido@gmail.com

\begin{abstract}
Resumen
Considerando que la actividad bibliotecaria mira por, en primer lugar, el tratamiento de la información, se observa que el profesional sabe, intuitivamente, la diferencia entre un texto científico y un texto narrativo de ficción pero sin mayores referencias acerca de los procedimientos específicos que se aplican a cada una de esas tipologías. Así, y considerando que la literatura tradicional del área de análisis documental dedica sus mayores esfuerzos al delineamiento de los procedimientos metodológicos aplicables al texto científico, cabe la pregunta: en términos conceptuales, ¿cómo puede ser definido un texto narrativo? Se puede afirmar que la diferenciación entre los textos reside en la estructura textual. En este sentido, se objetiva el acercamiento a metodologías para el Análisis Documental de Contenido que contemplen el texto narrativo de ficción. Para tal efecto, a partir de un texto de un autor contemporáneo, se aplica la clasificación del tema con la abstracción de conceptos, a través del Recorrido Generativo de Sentido, para la construcción de un índice, objetivando la indización de los conceptos. Como resultado, se obtuvo un conjunto de apoyos para una recuperación temática del texto narrativo de ficción, de una manera rápida y eficaz. Por ello, se puede concluir que el análisis documental de contenido de textos narrativos de ficción a través de la utilización del Recorrido Generativo de Sentido, se mostró válida.
\end{abstract}

Palabras clave: Texto narrativo. Análisis documental de contenido. Recorrido generativo de sentido. Epistemología. Metodología.

\section{Análisis documental de contenido}

\subsection{Elementos conceptuales}

El abordaje del análisis documental presupone el rescate del ciclo informacional como base para el hacerse documental, en cuyo ámbito se

\begin{abstract}
Considering that the library information treatment is basically devoted to subsidize research and leisure (differently from archival information treatment which is mostly devoted to guarantee the legal and proofvalue of information), it is possible to state that the information professional is able to, intuitively, distinguish a scientific text from a narrative text of fiction. In this way, and considering that the document analysis bigger efforts were dedicated in order to evidence metodological procedures suitable to scientific texts, which allows us to question in what extent can be defined a narrative text for subject analysis purposes. It can be affirmed, then, that the differentiation between the texts inhabits in the textual structure. In this sense, the aim is to establish methodologies to the subject analysis that can contemplate the narrative text of fiction. In this way, from a text of a contemporary author, it is applied the classification of the subject with the abstraction of concepts, by using the Generative Sense Course, for the construction of an index, objectifying the indexation of the concepts. As a result, it was obteined subsidies for a thematic recovery of the narrative text of fiction, in a fast and efficient way. In such a way, it can be concluded that the Content Analysis of narrative texts of fiction, through the use of the Gerative Sense Course, is revealed as feasible.
\end{abstract}

KeyWords: Narrative Text. Documentary Content Analysis. Generative Sense Course. Epistemology. Metodology.

verifican, como operaciones fundamentales e interdependientes, la producción, el tratamiento u organización, la recuperación, la diseminación y el uso de la información que, a su vez, podrá generar nueva producción, completando el ciclo. El tratamiento u organización de la información consiste, por lo tanto, en una etapa intermedia 
dirigida primordialmente a la garantía de un diálogo entre el productor y el consumidor de la información, asumiendo, de esa forma, una función de verdadero puente informacional.

La expresión análisis documental, cuyo desarrollo teórico floreció notadamente en las tradiciones francesa, española y brasileña, congrega dos conceptos fundamentales: análisis y documento, el primero relacionado a la descomposición de un contenido informacional en sus elementos constitutivos y, el segundo, entendido como información registrada, sea ella medio de prueba (tal como ocurre, por ejemplo, en el Derecho Procesal), materialización de un hecho (como en los documentos que revelan actos administrativos), soporte de información (tal como es tratado en la Catalogación, por ejemplo) o registro y base para generación de nuevos conocimientos (incorporando la idea de conocimiento registrado, como aborda el área de organización del conocimiento).

Analizándose distintas definiciones de análisis documental presentadas en la literatura internacional (1), se observan que los aspectos de identificación, extracción y representación de la información, pueden asumir una dimensión de forma o de contenido, de donde derivan las concepciones de análisis documental de forma y análisis documental de contenido, la primera ligada al proceso de descripción bibliográfica (catalogación) con el objetivo de crear registros $y$, la segunda, a los procesos de análisis y descripción de los aspectos intrínsecos del documento, ligados a su contenido temático, razón por la cual también se denomina tratamiento temático de la información.

En suma, se puede decir que el área de análisis documental de contenido, se materializa por medio de un conjunto de procedimientos de naturaleza analítico-sintética, envolviendo los procesos de análisis del contenido temático de los documentos y su síntesis, por medio de la condensación o de la representación en lenguajes documentales, con el objetivo de garantizar una recuperación rápida y precisa por el usuario o cliente. De esa concepción algunos elementos merecen destaque:

- procesos: el contenido del área se da por medio de una secuencia lógica de procedimientos;

- análisis: la descomposición de un todo en sus elementos constitutivos, buscando un sentido informativo;

- contenido temático: el conjunto de elementos documentales que reflejan la dimensión informativa (la función original) del documento;
- documentos: aquí entendidos en su concepción más amplia, como soportes informacionales de cualquier orden;

- condensación: reconstrucción del documento de forma abreviada, destacando sus puntos o pasajes de mayor expresividad temática;

- representación: proceso similar a la traducción, en el cual el contenido temático pasa a ser expreso de manera estandarizada conforme parámetros previamente establecidos;

- lenguajes documentales (también denominados lenguajes de indización): conjunto de instrumentos o herramientas para la representación estandarizada del contenido temático de los documentos.

- recuperación de la información: objetivo básico de toda la actividad de tratamiento documental, una vez que permite que el contenido informacional llegue hasta el usuario o cliente;

- rapidez: principalmente en tiempos de mucha producción informacional, es importante recordar que información retrasada se constituye, en verdad, en información negada;

- precisión: además de garantizar la rapidez, es fundamental que esa información llegue al usuario o cliente, en adecuación a las especificidades de su necesidad.

\subsection{Bases epistemológicas}

Las bases epistemológicas del análisis documental de contenido remontan a tres líneas teóricas que se construyeron y consolidaron históricamente, en distintas partes del mundo occidental.

Así, un primer abordaje se construyó a partir de la óptica del subject cataloguing (2), de orientación predominantemente norteamericana, y en buena parte norteada por los principios de la catalogación alfabética de Cutter y de la tradición de encabezamientos de asunto de la Library of Congress, cuyo énfasis reside en el catálogo como producto del tratamiento de la información en bibliotecas. Tal concepción es definida por Fiuza $(1985$, p.257) como "... representación, en los catálogos de biblioteca, de los asuntos contenidos en el acervo". En ese contexto, significativa contribución se observa, entre otros, en los trabajos de Cutter, Kaiser y Coates. En la actualidad, autores como la canadiense Hope Olson y el norteamericano Sanford Berman, entre otros, se han dedicado a los aspectos de la catalogación de asunto.

Un segundo abordaje, a su vez, se centró en la óptica del indexing (3), de orientación predomi- 
nantemente inglesa, en la cual los índices, como productos del tratamiento temático de la información, proceden de la utilización de lenguajes de indización, notadamente los tesauros, observándose una preocupación de naturaleza más teórica acerca de la construcción de tales lenguajes, en buena parte influenciada por los trabajos del Classification Research Group. En esa línea de pensamiento, se destacan, entre otros, los trabajos de Foskett, Austin, Farradane, Metcalfe, Aitchinson, Gilchrist y Lancaster (4).

Bajo otra dimensión, se tiene el abordaje de la analyse documentaire (5), de orientación predominantemente francesa (y con nítidos reflejos en la tradición científica española y brasileña), en la cual el enfoque se centra en el proceso de tratamiento temático en sí, vale decir, en la explicitación de los procedimientos dirigidos a la identificación y selección de conceptos para posterior representación y generación de productos. La referida línea presenta un fuerte interfaz con la Lógica, la Terminología y, en especial, la Lingüística, a partir de los trabajos pioneros de Coyaud y Gardin (6) y posee, como trazo distintivo, la búsqueda de una dimensión metodológica para el área, según la cual la definición y explicitación de procedimientos debe preceder necesariamente a la cuestión de los lenguajes de indización (como en la tradición inglesa) o de la generación de productos como los catálogos (tradición norteamericana).

A título de síntesis se puede entonces decir que el análisis documental de contenido comprende los siguientes procedimientos (Guimarães, 1994):

\section{Etapa analítica:}

- Lectura técnica del documento, en que el documentalista se adentra en la estructura del documento, buscando entrar en contacto con las partes que revelen mayor contenido temático valiéndose, para ello, de un conjunto de estrategias metacognitivas;

- Identificación de conceptos: una vez identificadas las partes más significativas temáticamente, se aplica al documento un conjunto de categorías conceptuales, mirando por la construcción de enunciados de asunto.

\section{Etapa sintética:}

- Selección de conceptos: los enunciados de asunto son, entonces, categorizados en principales, secundarios y periféricos, y ordenados lógicamente, teniendo como parámetros la estructura, la función y los usos (tipo de búsqueda informacional a que se presta el documento).
- Condensación documental: reducción del documento original a un micro-documento (resúmenes), y...

- Representación documental: traducción del contenido temático del documento al lenguaje de indización, representándolo por medio de índices.

Delante de lo expuesto, se observa que la construcción teórica del área de tratamiento temático de la información se desarrolló a partir del abordaje de tres aspectos - o facetas- que les son inherentes - los procesos, los productos e instrumentos-, tal como puede ser observado en la sistematización llevada a cabo en el VI Encuentro de Directores y Docentes de Biblioteconomía y Ciencia de la Información del MERCOSUR (Encontro..., 2002, p. 2):

\begin{tabular}{|c|c|c|c|}
\hline & \multicolumn{2}{|c|}{ Organización de la Información } & \multirow{2}{*}{$\begin{array}{l}\text { Recuperación } \\
\text { de la } \\
\text { Información }\end{array}$} \\
\hline & Forma & Contenido & \\
\hline 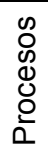 & $\begin{array}{l}\text { Catalogación } \\
\text { Control de } \\
\text { autoridades }\end{array}$ & $\begin{array}{l}\text { Análisis } \\
\text { Condensación } \\
\text { Representación }\end{array}$ & $\begin{array}{l}\text { Evaluación } \\
\text { Estrategias de } \\
\text { búsqueda }\end{array}$ \\
\hline $\begin{array}{l}\frac{0}{0} \\
\frac{0}{2} \\
\frac{0}{0} \\
0\end{array}$ & $\begin{array}{l}\text { Catálogos } \\
\text { OPAC's }\end{array}$ & $\begin{array}{l}\text { Índice } \\
\text { Resumen }\end{array}$ & \\
\hline 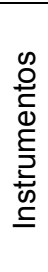 & $\begin{array}{l}\text { Formatos } \\
\text { Control de } \\
\text { autoridades }\end{array}$ & $\begin{array}{l}\text { Clasificaciones } \\
\text { Listas de } \\
\text { Encabezamiento } \\
\text { de Asunto } \\
\text { Tesauros } \\
\text { Terminologías } \\
\text { Ontologías }\end{array}$ & $\begin{array}{l}\text { Modelos de } \\
\text { recuperación } \\
\text { Sistemas de } \\
\text { RI } \\
\text { Interfaces }\end{array}$ \\
\hline
\end{tabular}

Cuadro 1: Sistematización de contenidos en organización y representación del conocimiento (Fuente: ENCONTRO..., 2002, p.2)

Como se puede observar, el universo del tratamiento temático de la información asumió una dimensión multifacetada, cuyos contenidos se construyeron, todavía, a partir de distintos diálogos con otras asignaturas tales como la Administración, la Diplomática, la Inteligencia Artificial, la Lingüística, la Lógica, la Psicología y la Terminología, entre otras, resaltándose, principalmente en los tiempos actuales, la naturaleza transversal (o instrumental) proveída por las nuevas tecnologías la información y la comunicación para el desarrollo - teórico y aplicado- del tratamiento temático de la información (7). 


\section{Consideraciones sobre la narración}

Considerando que la actividad bibliotecaria mira por, en un primer plan, el tratamiento de la información para fines de subsidiar la investigación y el solaz (diferentemente del tratamiento de la información archivística que subsidia primordialmente las actividades de naturaleza jurídico-probatoria), se observa que el profesional conoce, intuitivamente, la diferencia entre un texto científico y un texto narrativo de ficción. Sin embargo, ya hace mucho tiempo, la literatura del área de análisis documental ha resaltado la importancia de la desautomatización de procedimientos y de la substitución de los procesos intuitivos por procedimientos metodológicamente previsibles y defendibles.

Así, y considerando que la literatura tradicional del área de análisis documental ha dedicado sus mayores esfuerzos al delineamiento de los procedimientos metodológicos aplicables al texto científico, cabe la pregunta: en términos conceptuales, ¿cómo puede ser definido un texto narrativo?

A pesar del considerable número de trabajos dirigidos a la discusión de tipologías textuales (cf. Beaugrande, 1980; Van Dijk 1977; Fávero \& Koch, 1988; Adam, 1993; Marcuschi, 1996), las propuestas hasta ahora presentadas son apenas parcialmente convergentes $y$, a veces, cuando se trata de definir los criterios que dan sustentación a la tipología, enteramente divergentes.

Para la Semiótica, según Fiorin (1999), todos los textos tienen un nivel narrativo, si la narratividad es entendida como cualquier transformación de estado, pues todos, implícita o explicitadamente, trabajan con transformaciones.

Tomese un ejemplo que la teoría tradicional de los géneros no podría considerar como un texto narrativo: un teorema. Ese texto se articula en tres partes: el enunciado del teorema, la demostración y la afirmación de que la demostración se hace (q.e.d., quod erat demonstrandum). Cuando se hace la afirmación final, lo que se está diciendo es que, en el texto, se pasó de un estado de no demostrado a uno de demostrado.

Además, el debate no está limitado a los tipos, sino envuelve también los géneros textuales. Para Marcuschi (1996:7) los tipos o las categorizaciones son los modos básicos de organización de nuestra experiencia cotidiana. En este sentido, los tipos son también un medio de determinar las condiciones en que se formulan las expectativas que conducen la propia comprensión. El tipo posee una estructuración interna homogénea y raramente será encontrado en 'estado puro' en los textos de circulación social.
Considerando que los criterios adoptados por los diferentes autores conducen al establecimiento de varias tipologías, el análisis textual exige que se haga una opción. En ese sentido, se entiende que la propuesta de Van Dijk (1977; 1978) es la que, en la etapa actual de la reflexión, mejor abarca la estructura textual, y este estudio pasará a ser orientado por ese autor.

Van Dijk parte de la noción de superestructura. Para él, las superestructuras son estructuras globales que caracterizan un tipo de texto y no dependen del contenido; son la forma del texto. Son culturalmente adquiridas y tenidas como esquemas formales a los cuales el texto se adapta. Así, cuando se produce un texto, por ejemplo, una narrativa, hay un esquema previo a seguir; y cuando se lee y comprende un texto, también actúa el mismo esquema, que hace comprenderlo como una narrativa.

Todavía según Van Dijk (1989), subyacentes a las informaciones lingüísticas de la estructura de superficie, existen macroestructuras de organización en términos de categorías que funcionan como esquemas (frames) organizacionales almacenados en la memoria. A través de esos esquemas, se hace posible la reintegración de la información nueva a la previa y la reformulación de hipótesis. Constituye la forma lógica de un texto, el nivel cognitivo. Es el nivel del contenido, de los aspectos semánticos, en los cuales tema y tópico definen la representación del texto.

En el nivel superficial están las microestructuras que constituyen las proposiciones básicas del texto. En este nivel, es procesada la organización de la estructura lingüística. En la relación entre las proposiciones se da la coherencia del texto. A su vez, estrategias y procesos sintácticos que establecen relaciones entre esas proposiciones definen la cohesión textual y trazan la tesitura del texto. La microestructura, por lo tanto, es la estructura local de un texto, esto es, la estructura de las oraciones y su relación mutua de conexión y coherencia (Fávero \& Koch, 1988, p. 87).

Koch \& Fávero (1987) sugieren tres criterios para el establecimiento de una tipología: a) dimensión pragmática (macroactos de habla y actualización en situaciones comunicativas); b) dimensión esquemática global (superestructura de Van Dijk); c) dimensión lingüística de superficie (marcas sintáctico-semánticas). Con base en esos criterios, clasifican los textos en narrativos, descriptivos, expositivos o explicativos, argumentativos "stricto sensu", inyuntivos o directivos y predictivos.

Para cada uno de esos tipos se aplican las dimensiones pragmática, esquemática y lingüística 
de superficie. Considerando que, en ese trabajo, solo se consideran los textos narrativos, se explicitan a continuación, con base en Van Dijk y Koch \& Fávero (según organización sugerida por esas autoras, 1987, p. 5-6) los criterios de análisis de ese tipo textual:

\section{Narración:}

Superestructura: En la narrativa predominan las acciones. En la estructura clásica de la narrativa, la situación espacial y temporal, así como los personajes y los contextualizadores, son introducidos en el resumen; se siguen los acontecimientos, que abarcan la complicación, la evaluación y la resolución

Macroestructura: el tema abarca una persona, un ser animado, o una cosa definida antropológicamente. Presupone una idea de acción, de cambio de estado, de transformación o de acontecimiento. La secuencia temporal es fundamental.

Dimensión lingüística de superficie: predominan relaciones subordinativas, con un verbo de cambio en pasado e indicadores de tiempo y lugar.

Para confirmar estos criterios, véase la siguiente fábula de Millôr Fernandes:

\section{El Caballo y el Caballero}

1 Pues aunque parezca increíble, cuando el 2 hombre llegó a las puertas del cielo, San Pedro

3 dijo: "- ¡No puede entrar!"

4 "- ¿Cómo no puedo entrar? Tengo acta

5 notarial de buenos antecedentes y tengo buenos

6 antecedentes mismo."

7 "- Sé - respondió Saint Pierre - pero en el cielo

8 nadie entra sin caballo."

$9 \mathrm{Y}$ el hombre, no pudiendo argumentar con

10 Saint Peter, volvió. En el camino encontró un

11 viejo amigo y le preguntó a dónde iba. Dijo el

12 amigo que al cielo. Él le explicó entonces que,

13 sin caballo, "nada". El amigo entonces sugirió:

14 "Mira, San Pietro ya está viejo. Tú te quedas en

15 posición de cuadrúpedo, y monto en ti. Él no

16 percibe nada porque ya está viejo y miope y

17 nosotros entramos en el cielo."

$18 \mathrm{Y}$ así hicieron. En la puerta, el Santo miró al 19 nuestro héroe: "Ops, ¿usted de nuevo? Ah, con20 siguió caballo, ¿eh? Muy bien, amarre el caballo 21 ahí afuera y puede entrar."

MORALEJA: En el cielo no entran sujetos con ideas.

Se puede observar que todos los criterios fueron cumplidos, sea en el nivel de la superestructura (predominio de acciones con personajes); de la macroestructura (idea de acción y de cambio de estado); y de la dimensión lingüística de superficie (verbo de cambio en el pasado e indicadores de tiempo y lugar).

Confirmada la identificación del texto narrativo de ficción, se pasa a la etapa de proponer me- todologías para el análisis documental de contenido que contemplen este tipo específico de texto.

Para tanto, a partir de la fábula arriba, se buscó la clasificación del tema con la abstracción de conceptos, a través del Recorrido Generativo de Sentido, para la construcción de un índice, objetivando la indización de los conceptos.

Según Moraes y Guimarães (2006), la construcción del Recorrido Generativo de Sentido presupone tres rellanos: las estructuras fundamentales, las estructuras narrativas y las estructuras discursivas. En las estructuras fundamentales se encuentran las categorías semánticas que ordenan los contenidos del texto de manera más general y más abstracta; las estructuras narrativas pueden ser definidas como cambios de estado en términos de conjunción y disyunción (manipulación, competencia, performance y sanción); en el nivel de las estructuras discursivas aparecen las estructuras narrativas abstractas, las cuales pueden ser concretizadas a través de la figurativización o de la tematización, o sea, a través de temas o figuras.

Al analizar las estructuras narrativas y las estructuras discursivas, se obtuvo el siguiente resultado:

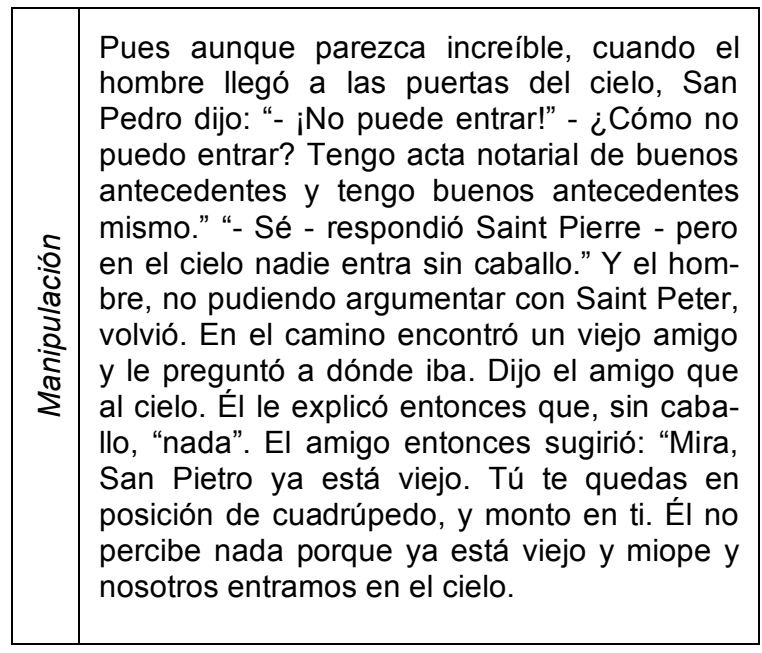

Terminado el proceso de levantamiento de las estructuras narrativas y el de las estructuras discursivas (temas y figuras), se procede a la construcción del recorrido temático y del recorrido figurativo para el levantamiento de temas. Según Moraes y Guimarães (2006), las figuras y los temas individualmente no poseen representatividad, a no ser a partir del momento en que se hace una conexión entre todos ellos, con el intuito de verificar cómo funcionan en el texto, 
trayendo en cuenta la cuestión de la isotopía como elemento amalgamador de sentidos.

\begin{tabular}{|l|l|}
\hline Temas & Figuras \\
\hline Buenos antecedentes & Puertas del cielo \\
\hline & No puede entrar \\
\hline & Caballo \\
\hline & Volvió \\
\hline & Amigo \\
\hline & Viejo \\
\hline & Miope \\
\hline & Entramos en el cielo \\
\hline
\end{tabular}

\begin{tabular}{|l|l|}
\hline Perfomance & $\mathrm{Y}$ así hicieron
\end{tabular}

\begin{tabular}{|l|l|}
\hline Temas & Figuras \\
\hline Hicieron & \\
\hline & \\
\hline & \\
\hline & \\
\hline
\end{tabular}

En la puerta, el Santo miró al nuestro héroe: "Ops, ¿usted de nuevo? Ah, consiguió caballo, ¿ ¿eh? Muy bien, amarre el caballo ahí afuera y क puede entrar."

œ MORALEJA: En el cielo no entran sujetos con ideas.

\begin{tabular}{|l|l|}
\hline Temas & Figuras \\
\hline No entran & Puerta \\
\hline & Santo \\
\hline & Caballo \\
\hline & Amarrar el caballo afuera \\
\hline & Cielo \\
\hline & Sujetos con ideas \\
\hline
\end{tabular}

En el texto en análisis, se puede percibir que las figuras "caballo" y "sujeto con ideas", al hacer el recorrido figurativo, adquieren el significado de "irracional" y "racional". Esta lectura es permitida al hacer el recorrido temático, finalizando con los temas "sagacidad" y "estupidez", y se hace una conexión con los temas del título, Caballo y Caballero.

De esta forma, a través de los recorridos temático y figurativo, se llega al tema del texto: sagacidad, confirmada por la estructura fundamental del texto, o elemento más abstracto, racional.

Al montarse un índice temático para indización, serían obtenidos los siguientes conceptos:

$$
\text { 1. Sagacidad Estupidez }
$$

\section{Racional Irracional}

Se puede percibir que el montaje reveló la relación entre términos antónimos, en los conceptos obtenidos.

\section{Consideraciones finales}

Como resultado, se obtuvieron apoyos metodológicos para una recuperación temática del texto narrativo de ficción, de una manera rápida y eficaz. De esta forma, se puede concluir que el Análisis Documental de contenido de textos narrativos de ficción, a través de la utilización del Recorrido Generativo de Sentido, se mostró ejecutable, en la medida en que proporcionó la clasificación del tema con la abstracción de conceptos para la construcción de un índice, cuya finalidad es la indización de los conceptos.

\section{Notas}

(1) Conjunto de operaciones necesarias para la extracción de la información contenida en las fuentes primarias para prepararla para su posterior recuperación y utilización (Ruiz Perez, 1992, p.51). Operación o conjunto de operaciones que sirven para representar el contenido de un documento bajo una forma distinta de su estado original, con el fin de facilitar la consulta o la posterior localización (Chaumier, 1993, p.17). Toda operación o grupo de operaciones que buscan la representación de un documento bajo una forma distinta de la original, sea por traducción, resumen o indización, de cara a facilitar la recuperación por expertos interesados (Gardin et alli, 1981, p.29). Técnica documental que permite, mediante una operación intelectual objetiva, la identificación y transformación de los documentos en productos que faciliten la consulta a los originales, en áreas de control documental, y con el objetivo último de servir a la comunidad científica (Garcia Gutierrez Apud Ruiz Perez, 1992, p.59). Proceso doble de identificación y representación del texto / documento (Pinto \& Galvez, 1996, p.31).

(2) Esa línea de abordaje ha encontrado, en periódicos como Cataloguing and classification quarterly, iniciado en 1980 y publicado por Harworth Press, un especial vehículo de divulgación, dedicado predominantemente a cuestiones de organización de registros bibliográficos y al control bibliográfico en general (www.cataloguingandclassification quarterly.com).

(3) Esa concepción ha encontrado en periódicos como The indexer, iniciado en 1958 y publicado por Society of Indexers, un especial vehículo de divulgación, dedicado predominantemente a cuestiones relativas a la historia, organismos, sistemas, estándares, métodos, prácticas y tecnología de indización. (www.theindexer.org).

(4) Con relación a las influencias sufridas por el área de tratamiento temático de la información, Pando (2005, p.103), rescatando el pensamiento de Bari- 
té (1998) considera que, a partir de las teorías y trabajos de Ranganathan, por medio de la aplicación de principios de la filosofía y de la lógica clásicas y el rigor de las ciencias matemáticas en la organización de campos conceptuales, se caracterizó, en el área, una reacción a la influencia pragmático-normalizadora norteamericana que, durante mucho tiempo permaneció como el modelo a ser utilizado en varios países. En Brasil, esa corriente, de origen inglés, tuvo especial influencia en importantes estudios e investigaciones llevados a cabo por distintos grupos de investigadores del área, notadamente de los programas de postgrado del IBICT y de la UnB.

(5) Aunque no se verifique, en la literatura, una revista que abrigue más nítidamente las discusiones de esa línea de abordaje, se percibe que los periódicos Documentaliste e Journal of Documentation, considerando su tradición más dirigida a la cuestión de la documentación, una mayor presencia de artículos ligados a las cuestiones metodológicas de análisis en sí.

(6) Tal corriente encontró reflejo en Brasil a partir de la década de 80, con los estudios del Grupo TEMMA, de la Escuela de Comunicaciones y Artes de la USP, coordinado por Johanna W. Smit. Igualmente en España esa concepción encontró reflejo, notadamente en los trabajos de Rafael Ruiz Perez (1992) y Maria Pinto Molina (Pinto Molina, 1993 y 1994, y Pinto \& Galvez, 1996).

(7) El aspecto de los diálogos interdisciplinares del área de tratamiento temático de la información así como el carácter transversal de las tecnologías de la información y de la comunicación ya fueron anteriormente abordados por este investigador (Guimarães, 2003 y Guimarães, 2004).

\section{Referencias}

Adam, J. M. Les textes: types et prototypes. Récit, description, argumentation et dialogue. Paris: Nathan, 1993.

Barité, M. Referenciales teóricos vigentes en el área de tratamiento temático de la información y su expresión metodológica. Porto Alegre: ABEBD, 1998. 7 p. [Relatório técnico do II Encontro de Dirigentes dos cursos superiores de Biblioteconomia dos países do Mercosul, Buenos Aires, 27-29 nov. 1997].

Beaugrande, R. Text, discourse, and process.London: Longman, 1980.

Chaumier, J. Indexação: conceito, etapas, instrumentos. Trad. José Augusto Chaves Guimarães. // Revista Brasileira de Biblioteconomia e Documentação, São Paulo, 21:1/2 (jan./jun.1988) 63-79.

Encontro de Diretores de Escolas de Biblioteconomia e Ciência da Informação, 6 . As articulações da pesquisa com o ensino e a extensão nos cursos de Biblioteconomia e Ciência da Informação no Mercosul: relato final. Londrina: ABECIN, 2002.

Fávero, L. \& I. Koch. Lingüística textual: introdução. São Paulo: Cortez, 1988

Fernandes, Millor. O cavalo e o cavaleiro. http://www2.uol. com.br/millor/fabulas/002.htm. Acesso en 02/03/2007

Fiorin, J.L. Sendas e veredas da semiótica narrativa e discursiva. // DELTA. 15:1 (fev./jul. 1999). http://www.sci elo.br/scielo.php?script=sci_arttext\&pid=S0102-4450 $1999000100009 \&$ Ing=pt\&nrm=iso>. ISSN 0102-4450.
Fiuza, M. M. O ensino de catalogação de assunto. // Revista da Escola de Biblioteconomia da UFMG. Belo Horizonte. 14:2 (set. 1985) 257-269.

Gardin, J. C. et al. La logique du plausible: essais d'épistemologie pratique. Paris : Ed. Maison des Sciences de l'Homme, 1981.

Guimarães, J. A. C. Análise documentária em jurisprudência: subsídios para uma metodologia de indexação de acórdãos trabalhistas brasileiros. São Paulo: Escola de Comunicações e Artes da USP, 1994. (Tese de doutorado em Ciências)

Guimarães, J. A. C. A análise documentária no âmbito do tratamento da informação: elementos históricos e conceituais. // Rodrigues, Georgete Medleg; Lopes, Ilza Leite. Organização e representação do conhecimento na perspectiva da Ciência da Informação. Brasília: Thesaurus, 2003.

Guimarães, J.A .C. Profissional da informação: desafios e perspectivas para sua formação. // Baptista, S. G.; Mueller, S. P. M. (org.). Profissional da informação: o espaço de trabalho. Brasilia: Thesaurus, 2004. 87-106.

Koch, I.; L. Fávero. Contribuição a uma tipologia textual. Letras e Letras, Uberlândia, 3:1 (1987) 3-10.

Marcuschi, L. A. Por uma proposta para a classificação dos gêneros textuais. Recife: UFPE, 1996.

Moraes, J. B. E.; Guimarães, J.A.C. Análisis documental de contenido de textos literarios narrativos: en busca del diálogo entre las concepciones de aboutness/meaning y de recorrido temático/recorrido figurativo. // Scire (Zaragoza), 2006. (No prelo).

Pando, Daniel Abraão. Formação e demanda profissional em tratamento temático da informação no Brasil: uma análise comparativa de conteúdos programáticos universitários e de concursos públicos em biblioteconomia. 2005. 187 f. Dissertação (Mestrado em Ciência da Informação) - Faculdade de Filosofia e Ciências, Universidade Estadual Paulista, Marília, 2005.

Pinto, M. Análisis documental: fundamentos y procedimientos. Madrid: EUDEMA, 1993.

Pinto, M. Interdisciplinary approaches to the concept and practice of written text's documentary content analysis (WTDCA). // Journal of documentation. 50:2 (June 1994) 111-133.

Pinto, M., Galvez, C. Análisis documental de contenido. Madrid: Sintesis, 1996.

Ruiz Perez, R. El análisis documental: bases terminológicas, conceptualización y estructura operativa. Granada : Ed. Universidad de Granada, 1992.

Van Dijk, T. La ciencia del texto. Barcelona: Paidós, 1989.

Van Dijk, T. Text and context. Explorations in semantics and pragmatics of discourse. London: Longman, 1977.

Van Dijk, T. The Porto Rico lectures on the structures and functions of discourse. Amsterdaá: 1978. 